\title{
Meta
}

Journal des traducteurs

Translators' Journal

\section{Modèles empiriques et expérimentaux en traductologie : question d'épistémologie}

\section{Jeanne Dancette et Nathan Ménard}

Volume 41, numéro 1, mars 1996

Le(s) processus de traduction / Translation Process(es)

URI : https://id.erudit.org/iderudit/002448ar

DOI : https://doi.org/10.7202/002448ar

Aller au sommaire du numéro

Éditeur(s)

Les Presses de l'Université de Montréal

ISSN

0026-0452 (imprimé)

1492-1421 (numérique)

Découvrir la revue

Citer cet article

Dancette, J. \& Ménard, N. (1996). Modèles empiriques et expérimentaux en traductologie : question d'épistémologie. Meta, 41(1), 139-156.

https://doi.org/10.7202/002448ar
Résumé de l'article

Les analyses sur les processus de traduction se situent dans un ensemble de recherches descriptives, expérimentales et théoriques qu'il convient d'examiner dans une perspective historique et critique. Cet éclairage épistémologique permet de mieux saisir la contribution de ces études et, en particulier, de l'analyse des processus, à la construction des modèles théoriques et explicatifs en traductologie. La présentation d'un exemple précis, extrait d'un protocole de verbalisation, offre aussi l'occasion de discuter des principaux problèmes qu'affrontent les traductologues dans leur tentative d'expliquer scientifiquement un phénomène aussi important que celui de la compréhension en traduction. 


\title{
MODÈLES EMPIRIQUES ET EXPERIMENTAUX EN TRADUCTOLOGIE : QUESTIONS D'ÉPISTÉMOLOGIE1
}

\author{
Jeanne Dancette et Nathan Ménard
}

Université de Montréal, Montréal, Canada

\begin{abstract}
Résumé
Les analyses sur les processus de traduction se situent dans un ensemble de recherches descriptives, expérimentales et théoriques qu'il convient d'examiner dans une perspective historique et critique. Cet éclairage épistémologique permet de mieux saisir la contribution de ces études et, en particulier, de l'analyse des processus, à la construction des modèles théoriques et explicatifs en traductologie. La présentation d'un exemple précis, extrait d'un protocole de verbalisation, offre aussi l'occasion de discuter des principaux problèmes qu'affrontent les traductologues dans leur tentative d'expliquer scientifiquement un phénomène aussi important que celui de la compréhension en traduction.
\end{abstract}

\begin{abstract}
Research on translation processes is part of descriptive, experimental and theoretical studies that need to be examined from a historical and critical standpoint. An epistemological perspective helps to assess the contribution of such studies - in particular processoriented ones - to the construction of theoretical and explanatory models in translation. This article includes a segment of think-aloud protocol, which leads to a discussion of the main problems researchers have in providing scientific explanations of the comprehension process in translation.
\end{abstract}

\section{INTRODUCTION}

L'objet de cet article est de porter un regard épistémologique sur les études empiriques consacrées au processus de traduction afin d'évaluer leur contribution à l'établissement de la traductologie comme discipline. Dans un survol rapide des principaux types de recherches empiriques, nous dégagerons les étapes nécessaires d'une démarche scientifique (parmi d'autres possibles) : du stade élémentaire mais indispensable de la collecte des données et de la description des phénomènes observés, au stade plus avancé de l'élaboration de modèles à valeur explicative. $\grave{A}$ ce deuxième stade, on reconnaît habituellement deux démarches: la démarche inductive qui sous-tend les généralisations à partir des données d'observation et la démarche hypothético-déductive par laquelle des propositions issues de constructions théoriques peuvent être soumises à une validation logique ou expérimentale.

Cherchant à apprécier l'apport théorique de telles recherches, nous mentionnerons certains des postulats, hypothèses et positions de nature linguistique, psycholinguistique et pragmatique sur lesquels reposent les études empiriques. Dans la dernière partie de cet article, nous prendrons, à titre d'illustration, les questions particulières du sens et de l'interprétation afin de montrer la valeur explicative des modèles expérimentaux, tant pour la traductologie que pour leur contribution à une théorie du langage. Pour ce faire, nous nous appuyons sur l'analyse d'un protocole de verbalisation pour en montrer la portée et les limites. Notre réflexion épistémologique sur les modèles théoriques est 
guidée par la synthèse critique, mais modérée, de la philosophie des sciences que propose Chalmers (1987).

\section{LES ÉTUDES EXPÉRIMENTALES ET LEUR APPORT THÉORIQUE À LA TRADUCTOLOGIE}

On distingue généralement en sciences humaines deux grandes catégories de recherches empiriques, qui, dans certaines limites, peuvent être combinées: les études quantitatives basées sur des études de corrélations et celles, qualitatives, qui reposent sur l'observation d'une situation ou d'un phénomène en train de se dérouler, dans son cadre habituel ou dans un cadre expérimental. Il y aurait une troisième catégorie, qui relève peut-être plus de l'informatique que des sciences humaines, celle de la simulation cybernétique du processus, comme par exemple la TA et la TAO et l'I.A. Nous la laisserons de côté dans le cadre de cet article qui porte sur l'observation des processus humains.

\section{1. Études quantitatives}

Pour faciliter la discussion, distinguons deux types d'études quantitatives en traduction : 1) celles qui portent sur l'analyse (généralement dans une optique comparatiste) des composantes linguistiques des textes source et cible, en neutralisant autant que possible la variable liée au traducteur ; 2) celles qui s'attachent à l'étude de variables qui influencent le déroulement d'un événement dont les agents sont les individus pris comme représentants d'une population cible pour l'étude du phénomène visé.

Dans la première catégorie, mentionnons l'étude de la cohésion en traduction (Bélanger 1992), ou de la charnière (Demers 1992). Dans la deuxième catégorie, nous relevons au Canada l'étude longitudinale de C. Séguinot et C. Klein-Lataud (Séguinot 1990) effectuée sur 151 étudiants de traduction auxquels ont été administrés trois tests, en $1^{\text {re }}$ et en $4^{\mathrm{e}}$ année de leur programme, et celle de Dancette (1995) sur l'analyse de corrélations entre les résultats obtenus par 20 étudiants de maîtrise en traduction (dont 4 professionnels) à quatre épreuves de nature différente visant à vérifier leurs connaissances linguistiques, thématiques, leur compréhension de texte et l'adéquation sémantique de leur traduction.

On pourrait souhaiter que les études quantitatives soient plus nombreuses, plus régulières et plus systématiques. D'une part, elles apportent plus de rigueur aux analyses en forçant l'élaboration de définitions plus fines des catégories et des éléments sur lesquels portent les recherches, par exemple, classes de relations anaphoriques chez Bélanger (1992). D'autre part, la méthode et les conclusions font voir la nature complexe de phénomènes jusque là traités comme des entités simples et remettent souvent en question des idées reçues, par exemple, l'idée selon laquelle le français serait une langue plus articulée syntaxiquement que l'anglais (Demers 1992). Mieux encore, elles font voir les difficultés que pose dans la pratique la reconnaissance d'entités considérées comme discrètes et qui, en fait, sont de nature continue (par ex. : les unités de traduction). C'est le cas aussi pour tout ce qui touche de près ou de loin aux structures sémantiques : y a-t-il une nette distinction entre les structures linguistiques et les structures extralinguistiques ?

Quant aux études portant sur des individus, elles sont très utiles pour dégager de façon synthétique les tendances observables dans un groupe cible considéré comme un tout, que ce soit un groupe d'étudiants de traduction ou de langues, ou encore de professionnels, et que l'on situe l'étude sur l'axe synchronique ou diachronique. Un des principaux défis est de rendre compte de la variabilité des comportements et des compétences entre sujets ou chez le même sujet.

Indépendamment de leur mérite du point de vue descriptif, la valeur explicative de ces études est faible et cela pour deux raisons principales. En premier lieu, la portée des 
résultats issus d'études statistiques est souvent limitée soit par la représentativité discutable des corpus, soit, en ce qui concerne les processus, par la trop petite taille des groupes témoins. Un groupe d'une soixantaine de sujets est généralement considéré en études linguistiques comme un minimum pour segmenter en sous-catégories $(\mathrm{N}=17$, deux variables) et pour établir des corrélations statistiquement fiables (Muller 1973; Ménard 1983). Or, pour des raisons pratiques, il est difficile, d'une part, de soumettre 60 personnes à des tests de traduction et de connaissances qui peuvent durer plus de deux heures et, d'autre part, le traitement d'un corpus aussi considérable aux fins de codage, d'évaluation et d'analyse est extrêmement coûteux en temps, en effort et en argent.

La seconde limite est fondamentale en regard de l'interprétation des résultats. L'observation d'une corrélation forte entre deux variables n'autorise nullement l'analyste à établir avec assurance des liens de cause à effet entre un événement et un autre, mais seulement à en poser l'hypothèse. Par exemple, une corrélation forte entre l'expérience professionnelle et l'adéquation sémantique de la traduction, telle qu'observée dans Dancette (1995), ou, dans le cas de Séguinot (1990), l'absence de corrélation pour certaines habiletés entre la formation académique et la qualité de la traduction, au mieux renforce, au pire infirme, mais ne suffit pas à démontrer l'hypothèse d'une relation de cause à effet entre les deux événements. Il faut tenir compte, en effet, de l'influence possible d'autres variables sur l'établissement de cette corrélation, par exemple, la familiarité avec le sujet, la motivation du traducteur, la situation pragmatique, etc.

L'analyse des données statistiques conduira donc souvent à la formulation de nouvelles hypothèses comme, par exemple, l'effet d'un seuil de connaissances minimales pour que les compétences traductionnelles et linguistiques soient mises à profit, $c f$. Dancette (1995). On peut aussi postuler de l'étude de Séguinot (1990), même si l'auteur ne le fait pas explicitement, l'existence d'un seuil d'intégration des enseignements pour que les compétences linguistiques et traductionnelles se reflètent dans une amélioration de la qualité des traductions des étudiants. La vérification des nouvelles hypothèses et l'exploration des relations explicatives entre les variables devront alors faire l'objet d'études qualitatives.

\section{2. Études qualitatives du processus}

Basées sur l'observation, elles s'attachent à décrire le comportement des sujets pris individuellement, en train d'accomplir une tâche. Elles ciblent non plus le résultat de la tâche mais le processus mental par lequel la tâche est accomplie.

Les méthodes empiriques basées sur l'observation du processus «sur le vif» se sont développées dans les années 1980 (parution en 1982 de la thèse de U. Sandrock, suivie de très nombreuses publications sur le sujet, en Allemagne surtout). Elles s'inscrivent dans la lignée des nombreuses études de psycholinguistique et de psychologie cognitive qui portent sur une grande variété d'opérations intellectuelles : problèmes de mathématiques, jeu d'échec, rappel d'histoires, apprentissage de la lecture chez les enfants, acquisition de la langue seconde, précis-writing, capacité à porter des jugements métalinguistiques sur un texte, et, bien sûr, traduction.

En traduction, les objectifs sont multiples et on constate une certaine évolution. Les premières études avaient pour principal objet la construction du modèle analytique : décomposition des opérations intellectuelles qui définissent le processus de traduction, identification de l'unité de traduction, classement des données sur lesquelles portent ces opérations (Sandrock 1982; Dechert et Sandrock 1986; Dechert 1987; Krings 1986; Gerloff 1987 ; Lörscher 1991, entre autres). Les études ultérieures, tout en intégrant ces acquis, ont pu consacrer plus d'efforts à l'approfondissement des dimensions psychologique et cognitive du processus : conditions psychologiques de la créativité (Kussmaul 1995), engagement émotif face au thème traité (Tirkkonen-Condit 1992), attention 
(Jääskeläinen 1993), recours aux connaissances thématiques (Schäffner 1993 et Dancette 1994) ou aux connaissances grammaticales (Mondahl et Jensen 1992). Cette évolution a permis de porter davantage attention à l'analyse des circonstances de la mise en œuvre du processus et à l'identification des conditions nécessaires ou suffisantes pour son bon déroulement.

La méthode la plus utilisée est celle qui s'appuie sur les protocoles de verbalisation, ou méthode du raisonnement à voix haute (think-aloud protocol). Elle consiste à demander au traducteur d'exprimer à voix haute toutes les pensées qui lui viennent à l'esprit dans l'exécution de sa tâche, autant les réactions émotives face à un aspect du travail (expressions de frustration ou de satisfaction) que les prises de conscience à propos des opérations et des raisonnements auxquels il se livre. Plusieurs chercheurs ajoutent à cette méthode d'enregistrement sur magnétophone l'enregistrement sur vidéo (Gerloff 1988 ; Séguinot 1989 ; Dancette 1994) qui permet d'obtenir des informations sur les gestes réellement posés (dictionnaire consulté à telle entrée, soulignements ou raturages dans le texte, etc.) et sur la durée des opérations. Parfois, des entrevues ou l'administration ultérieure de questionnaires complètent la collecte des données obtenues grâce aux protocoles verbaux.

Il y a cependant des limites théoriques et méthodologiques à ces approches d'enregistrement du sujet sur le vif : nature très fragmentaire de la verbalisation et probabilité de grandes distorsions entre le processus réel (boîte noire) et la verbalisation. Ces limites sont abondamment discutées, entre autres, par Krings (1986) et Lörscher (1991).

Indépendamment de ces limites, l'apport des recherches sur les protocoles à la compréhension des opérations de traduction est manifeste, non seulement par l'enrichissement du cadre descriptif mais aussi par la valeur explicative des positions théoriques. Cette nouvelle dimension entraîne un élargissement des débats et introduit les exigences de prudence et d'évaluation critique face aux positions, aux méthodes et aux résultats.

\subsection{Signification épistémologique des recherches sur les processus}

\subsection{1. Établissement de consensus}

Il est intéressant de relever quelques-uns des postulats sur lesquels il semble y avoir consensus : a) la globalité de l'acte traduisant, b) la nature relationnelle de cet acte, due à la dépendance des éléments les uns des autres (phrase, texte, environnement pragmatique) et c) la très grande variabilité du processus de traduction d'un sujet à un autre, d'un moment à un autre ou d'un texte à un autre chez le même sujet.

\subsubsection{Exemples d' hypothèses}

De ces postulats est issue une série d'hypothèses et de positions théoriques sur lesquelles se rencontre l'ensemble des chercheurs. Notons quelques exemples.

Directionnalité du processus de traduction. L'hypothèse du mouvement de va et vient entre les différents niveaux de lecture avait déjà été posée à l'encontre d'une conception linéaire de la traduction. La direction préférentielle que suit le processus est exprimée par l'opposition entre les stratégies descendantes et ascendantes (top down vs bottom up) discutées par Kussmaul (1995), entre autres, ou globales vs locales (Jääskeläinen 1993) ou sense-oriented vs form-oriented (Lörscher 1991) ou centripètes vs centrifuges (Dancette 1995), et par le concept de compensation (stratégies de complémentarité et de supplétion entre les différents niveaux de traitement cognitif, $c f$. Dancette 1995).

Rôle des connaissances extralinguistiques et leur relation avec le linguistique. Les études expérimentales sur les processus de traduction avaient permis de mettre 
à l'épreuve et de préciser les positions théoriques qui jusqu'alors n'avaient pas été vérifiées dans la pratique, notamment celle de la déverbalisation selon laquelle «les formes linguistiques de l'original s'évanouissent pour ne laisser subsister que la conscience du sens» (Seleskovitch $1984: 85$ ) et celle de la place des connaissances du monde, gardées dans la mémoire à long terme. Il s'agit surtout de la théorie sur les «cadres de connaissances» de Minsky (1974) et de Fillmore (1976), reprise par Kussmaul (1995) et Dancette (1995).

Il faut aussi mentionner certaines suppositions pragmatiques et intuitives sur lesquelles il n'y a pas forcément consensus, par exemple, en ce qui concerne le rôle des variables qui devraient être en étroite corrélation. Krings (1988) veut vérifier l'hypothèse selon laquelle les traducteurs professionnels démontreraient dans leur protocole de verbalisation le recours à un plus grand nombre d'opérations traductionnelles de routine que les traducteurs non professionnels. Lörscher (1992) part de l'hypothèse selon laquelle les professionnels auraient des stratégies traductionnelles plus orientées vers le sens (senseoriented) que vers la forme (form-oriented) à la différence du traducteur non professionnel. De tels résultats ne nous surprendraient pas. Toutefois, contrairement aux attentes, Gerloff (1988) et Jääskeläinen (1993) constatent qu'expérience professionnelle, d'une part, et connaissances linguistiques, d'autre part, ne sont pas toujours en corrélation avec la qualité de la traduction.

De telles conclusions, contradictoires, paradoxales ou particulières, indiquent qu'il est nécessaire de poursuivre les recherches afin d'explorer le rôle de variables plus fines qui contribueraient à une meilleure explication des phénomènes.

\subsubsection{Progrès méthodologiques}

Sans minimiser les problèmes posés par les différences entre chercheurs quant aux méthodes et à la terminologie, on conviendra que les paramètres d'analyse des protocoles (Krings 1986), de classification des stratégies (Lörscher 1991) ou même les critères de définition des concepts fondamentaux tels que stratégie, processus, comportement, forment déjà une base pour la reprise des mêmes outils méthodologiques dans d'autres expériences et par d'autres chercheurs.

Les grilles d'analyse, les schémas et les modèles marquent une étape par rapport à la simple description des données expérimentales. Ils rendent possible la reprise des expériences et l'uniformité des méthodes de traitement des données. Ils peuvent augmenter le rendement de la recherche expérimentale en raison du caractère cumulatif des données et des résultats. La répétition des expériences est, en effet, une condition primordiale pour confirmer la validité d'un modèle, tester sa valeur explicative et pour synthétiser les résultats au moyen de formalisations.

Ainsi, le modèle de Lörscher (1991) compte 22 éléments de stratégies qu'il regroupe en des structures plus simples constituées de chaînes d'opérations stratégiques. On peut répéter l'expérience en appliquant le modèle à plusieurs groupes de traducteurs, étudiants ou professionnels, enfants ou adultes bilingues. Mentionnons également la possibilité de comparer les résultats avec ceux obtenus par des recherches analogues avec des contraintes psycholinguistiques de même ordre, par exemple, sur les stratégies de communication chez les apprenants adultes de langue seconde, ou les processus de lecture et d'écriture en langue maternelle ou en langue seconde (Hartman 1991; Grant 1992 ; Whalen et Ménard 1995).

\subsubsection{Contribution à des débats plus généraux}

Les protocoles de verbalisation et les outils méthodologiques de même nature revêtent un intérêt d'autant plus grand qu'ils s'inscrivent dans une démarche théorisante plus ou moins clairement énoncée, reposant sur des hypothèses de nature linguistique, 
psycholinguistique et pragmatique. Les hypothèses mentionnées ci-dessus ne sont pas toutes spécifiques à la traduction, mais, du fait que la traduction offre un terrain d'expérimentation très favorable à l'étude du fonctionnement du langage humain, elles peuvent être soumises à une vérification. De ce point de vue, les recherches empiriques sur les processus de traduction s'accompagnent des débats théoriques généraux issus des diverses disciplines voisines, en linguistique, en sciences cognitives, en pragmatique. Parmi ces débats soulevant des questions non résolues, nommons celles de la définition du sens contextuel, de la place du linguistique dans le processus de lecture et d'interprétation, de l'existence d'une frontière entre le linguistique et l'extralinguistique, etc.

Il est donc normal que les chercheurs soient tentés de dépasser le stade élémentaire de la collecte et de la description de données empiriques, de passer du niveau phénoménologique (qui relie le processus au produit) au niveau explicatif, et du niveau exploratoire à celui de la généralisation. La contribution à l'établissement d'une théorie suppose en effet un tel développement.

Dans une perspective de réflexion épistémologique, il est intéressant de noter que le modèle d'analyse des processus permet d'atteindre un double objectif et peut servir non seulement à un examen des modèles descriptifs, mais aussi à circonscrire la problématique dans la construction des théories traductologiques. Nous allons, dans la deuxième partie de cet article, reproduire un exemple de protocole verbal et en faire l'évaluation critique sous ce double éclairage.

\section{2. ÉYALUATION CRITIQUE D'UNE GRILLE D'ANALYSE DE PROTOCOLES DANS UNE PERSPECTIVE THÉORIQUE}

\subsection{Présentation du cas}

Nous reprenons une partie d'une expérience conduite auprès de cinq étudiants, dont deux sont des traducteurs professionnels. Ils devaient verbaliser les opérations qu'ils effectuaient dans le cadre de leur tâche de traduction. La méthode, exposée dans Dancette (1994 et 1996 à paraître), est fondée sur l'analyse des protocoles verbaux (audio et vidéo), de la traduction produite par les répondants et de leurs réponses à un questionnaire vérifiant les connaissances extralinguistiques liées à des segments du texte.

Texte à traduire : (...) Once the victim of price wars, Compaq is now the aggressor. Its low-cost ProLinea PCs, introduced last June, were an immediate hit, spurring industrywide price cuts and generating a three-month order backog.

Ce passage est la deuxième phrase d'un article extrait de la revue Business Week. Il était soumis en entier aux traducteurs. La première partie est reproduite en annexe.

Dans le tableau qui suit, nous avons retranscrit verbatim le protocole de verbalisation d'un des répondants correspondant à son travail sur le sens et la traduction de l'expression three-month order backlog à laquelle il consacre 25 minutes. Notons que ce répondant a une expérience professionnelle de la traduction et de l'interprétation d'environ deux ans qui s'ajoute à sa formation académique dans le cadre de la maîtrise en traduction de l'Université de Montréal.

La grille comporte le protocole lui-même et, dans les colonnes de gauche et de droite, des éléments permettant d'analyser ce protocole : 1) la nature de l'opération effectuée (lecture, recherche, décodage, etc.) ; 2) le locus ou le focus d'attention (définis comme l'unité de texte, du texte source ou du texte cible, qui déclenche l'opération); 3 ) le niveau d'analyse où se situe l'opération. Des conventions de codage des différentes opérations sont proposées dans Dupont (1993).

Traduction écrite du répondant (avec ses propres ratures et les deux versions successives) : 
Hier victime de la guerre des prix, Compaq est aujourd' hui prédateur. Grâce à son cồt peu élevé faible coût, l'ordinateur personnel Prolinea, lancé sur le marché en juin dernier, a connu un succès fulgurant,

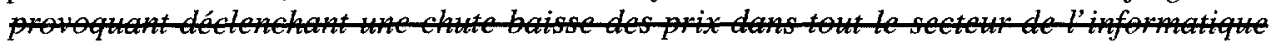
et provoquant un retard de trois mois dans la livraison des commandes et débouchant sur une baisse des prix dans le secteur de l' informatique.

\section{tor}

Hier encore victime de la guerre des prix, Compaq est devenu prédateur: Grâce au coût peu élevé de l'ordinateur personnel Prolinea, lancé sur le marché en juin dernier, l'entreprise a connu un succès fulgurant provoquant un retard de trois mois dans la livraison des commandes et déclenchant une baisse des prix dans le secteur de l'informatique.

\begin{tabular}{|c|c|c|c|c|}
\hline & $\begin{array}{l}\text { Nature de } \\
\text { l'opération }\end{array}$ & Protocole de verbalisation & Locus / Focus & $\begin{array}{l}\text { Niveau } \\
\text { d'analyse }\end{array}$ \\
\hline 1 & $\begin{array}{l}\text { Définition } \\
\text { empirique }\end{array}$ & $\begin{array}{l}\text { «Backlog», c'est des commandes mais des } \\
\text { commandes en retard... }\end{array}$ & backlog & $\begin{array}{l}\text { lexico- } \\
\text { sémantique }\end{array}$ \\
\hline 2 & $\begin{array}{l}\text { Reconnaît } \\
\text { problème } \\
\text { par réaction } \\
\text { émotive }\end{array}$ & «backlog» ha ! je déteste ce mot là ... & backlog & méta-lexical \\
\hline 3 & $\begin{array}{l}\text { Consulte } \\
\text { Rob\&Coll., } \\
\text { réévalue } \\
\text { méthode de } \\
\text { repérage dans } \\
\text { le dictionn. }\end{array}$ & $\begin{array}{l}\text { B, C, ho ! je vais pas lire tout ça, flûte ! } \\
\text { «backroom, background», voyons dans les } \\
\text { compounds ... verbe intransitif, j'en ai rien } \\
\text { à fiche ... Ah voilà ! Compound ... } \\
\text { backbencher, backbiting, backfire, } \\
\text { backhanded, backlash, }\end{array}$ & backlog & $\begin{array}{l}\text { lexico- } \\
\text { sémantique }\end{array}$ \\
\hline 4 & $\begin{array}{l}\text { Trouve } \\
\text { nouvelle } \\
\text { définition }\end{array}$ & $\begin{array}{l}\text { backlog ! le voilà ... un arriéré de travail, } \\
\text { ah! une accumulation ... }\end{array}$ & backlog & $\begin{array}{l}\text { lexico- } \\
\text { sémantique }\end{array}$ \\
\hline 5 & $\begin{array}{l}\text { Confirme } \\
1^{\text {re }} \text { définition } \\
\text { empirique }\end{array}$ & c'est bien ce que je pensais. & backlog & $\begin{array}{l}\text { lexico- } \\
\text { sémantique }\end{array}$ \\
\hline 6 & $\begin{array}{l}\text { Enrichit la } \\
\text { définition, } \\
\text { explication } \\
\text { contextuelle } \\
\text { par inférence }\end{array}$ & $\begin{array}{l}\text { Oui. Mais, un arriéré de travail ... ça veut } \\
\text { dire qu'en fait il y a plus de commandes } \\
\text { que le producteur peut fournir, commandes } \\
\text { supérieures à l'offre ... }\end{array}$ & backlog & $\begin{array}{l}\text { sémantique } \\
\text { textuelle }\end{array}$ \\
\hline 7 & Traduit & dans tous les secteurs de l'informatique. & industry-wide & syntagmatique \\
\hline 8 & Lit & $\begin{array}{l}\text { Ah la la ! Voila que je lis la suite } \\
\text { maintenant ... }\end{array}$ & article & procédural \\
\hline 9 & $\begin{array}{l}\text { Recherche } \\
\text { contexte et } \\
\text { co-texte } \\
\text { (pays } \\
\text { d'origine de } \\
\text { l'article) }\end{array}$ & $\begin{array}{l}\text { «Undercutting market leader» OK «then it } \\
\text { extended the price battle on the US with } \\
\text { cut-rate» Bon, alors, in the US. Ou on se } \\
\text { retrouve? Apparemment au Canada, oui } \\
\text { Business Week ça peut être ça ... }\end{array}$ & $\begin{array}{l}\text { Bus. Week-> } \\
\text { US, Canada. }\end{array}$ & $\begin{array}{l}\text { intratextuel/ } \\
\text { extra- } \\
\text { linguistique }\end{array}$ \\
\hline 10 & $\begin{array}{l}\text { Consulte } \\
\text { graphique } \\
\text { de l'article }\end{array}$ & Voyons le «chart» & graphique & intratextuel \\
\hline
\end{tabular}




\begin{tabular}{|c|c|c|c|c|}
\hline & $\begin{array}{l}\text { Nature de } \\
\text { l'opération }\end{array}$ & Protocole de verbalisation & Locus / Focus & $\begin{array}{l}\text { Niveau } \\
\text { d'analyse }\end{array}$ \\
\hline 11 & $\begin{array}{l}\text { Explique } \\
\text { et paraphrase }\end{array}$ & $\begin{array}{l}\text { «lower prices ... should restore», oui, c'est } \\
\text { bien ça. Ça a permis une chute des prix, une } \\
\text { dégringolade des prix ... mais c'est pas } \\
\text { dégringolade parce qu'on a l'impression } \\
\text { que c'est les prix qui ont volontairement } \\
\text { fait ça, alors qu'il y a une décision dans le } \\
\text { secteur de l'informatique de réduire les prix } \\
\text { justement. Une baisse des prix tout } \\
\text { simplement ... dans tout le secteur de } \\
\text { l'informatique et provoquant }\end{array}$ & lower prices & $\begin{array}{l}\text { interproposi- } \\
\text { tionnel, } \\
\text { (logique des } \\
\text { mondes } \\
\text { possibles) }\end{array}$ \\
\hline 12 & $\begin{array}{l}\text { Constate } \\
\text { problème, } \\
\text { indice émotif }\end{array}$ & $\begin{array}{l}\text { Me casse les pieds, ce «backlog» ... «order } \\
\text { backlog» ... }\end{array}$ & backlog & lexical \\
\hline 13 & Traduit & $\begin{array}{l}\text { un retard de trois mois dans l'exécution des } \\
\text { commandes ... }\end{array}$ & $\begin{array}{l}\text { (order)backlog } \\
\text {... }\end{array}$ & $\overline{\text { syntagmatique }}$ \\
\hline 14 & $\begin{array}{l}\text { Contrôle, } \\
\text { révise } \\
\text { cooccurrents } \\
\text { lexicaux }\end{array}$ & $\begin{array}{l}\text { Non, on n'exécute pas une commande, on } \\
\text { remplit une commande... }\end{array}$ & $\begin{array}{l}\text { collocations } \\
\text { verbales de } \\
\text { commande }\end{array}$ & phraséologique \\
\hline 15 & $\begin{array}{l}\text { Consulte } \\
\text { Petit Robert. } \\
\text { Recourt aux } \\
\text { contextes } \\
\text { dictionn. } \\
\text { pour préciser } \\
\text { sens par } \\
\text { élimination }\end{array}$ & $\begin{array}{l}\text { Allez ... commande ... livrer, voilà : livrer } \\
\text { une commande, exécuter une commande, } \\
\text { on exécute un travail sur commande ... } \\
\text { mm... }\end{array}$ & idem & $\begin{array}{l}\text { lexico- } \\
\text { sémantique }\end{array}$ \\
\hline 16 & $\begin{array}{l}\text { Consulte } \\
\text { Petit Robert } \\
\text { Paraphrase, } \\
\text { explication }\end{array}$ & $\begin{array}{l}\text { Un retard de trois mois dans les livraisons? } \\
\text { puisque, pour que le travail soit livré, il } \\
\text { doit avoir été exécuté. Voyons alors, ... } \\
\text { faire passer une commande ... livrer une } \\
\text { commande ... commande, cordage, on s'en } \\
\text { fout ... télé commande, tenir les commandes } \\
\ldots \text {... réglage ... la commande et la réponse ? } \\
\text { Bon alors, c'est livrer ... un retard de trois } \\
\text { mois dans les livraisons, dans la ... }\end{array}$ & $\begin{array}{l}\text { three-month } \\
\text { order backlog }\end{array}$ & $\begin{array}{l}\text { extraling. } \\
\text { (logique des } \\
\text { mondes } \\
\text { possibles) }\end{array}$ \\
\hline 17 & $\begin{array}{l}\text { Hésite, met } \\
\text { en attente } \\
\text { problème } \\
\text { non résolu }\end{array}$ & $\begin{array}{l}\text { Backlog, c'est ça, dans les livraisons ... } \\
\text { Est-ce que c'est ça? }\end{array}$ & backlog & $\begin{array}{l}\text { lexico- } \\
\text { sémantique }\end{array}$ \\
\hline 18 & $\begin{array}{l}\text { Explore } \\
\text { lien de } \\
\text { dépendance } \\
\text { syntaxico- } \\
\text { sémantique } \\
\text { entre mots }\end{array}$ & $\begin{array}{l}\text { En plus, c'est pas clair. Est-ce que c'est } \\
\text { «industry-wide»? Non, le «industry-wide», } \\
\text { c'est les «price cuts», }\end{array}$ & $\begin{array}{l}\text { order backlog } \\
\text { phrase }\end{array}$ & $\begin{array}{l}\text { textuel } \\
\text { (relations } \\
\text { thématiques } \\
\text { price } \\
\text { cut/Compaq) }\end{array}$ \\
\hline 19 & idem & $\begin{array}{l}\text { mais le «backlog», c'est chez, j'ai } \\
\text { l'impression que c'est chez Compaq. }\end{array}$ & $\begin{array}{l}\text { order backlog } \\
\text { phrase }\end{array}$ & $\begin{array}{l}\text { textuel } \\
\text { (relations } \\
\text { thématiques } \\
\text { backlog/ } \\
\text { Compaq) }\end{array}$ \\
\hline
\end{tabular}




\begin{tabular}{|c|c|c|c|c|}
\hline & $\begin{array}{l}\text { Nature de } \\
\text { l'opération }\end{array}$ & Protocole de verbalisation & Locus / Focus & $\begin{array}{l}\text { Niveau } \\
\text { d'analyse }\end{array}$ \\
\hline 20 & Relit & $\begin{array}{l}\text { «They were an immediate hit spurring ... } \\
\text { and generating a three-month order } \\
\text { backlog»..., }\end{array}$ & $\begin{array}{l}\text { order backlog } \\
\text { phrase }\end{array}$ & textuel \\
\hline 21 & $\begin{array}{l}\text { Confirme } \\
\text { lien de } \\
\text { dépendance } \\
\text { établi en } 19\end{array}$ & \begin{tabular}{|l|} 
ça peut pas être à l'échelle de industry. \\
C'est ça, moi, j'inverserais plutôt, parce que \\
j'ai l'impression que le retard de trois mois \\
dans les livraisons, c'est parce qu'ils ont eu \\
un surplus de commandes, parce que c'était \\
un grand succès ... C'était un tel succès ... \\
et ils ont eu tellement de commandes qu'à \\
ce moment là on peut passer du particulier, \\
donc de Compaq, enfin, au secteur de \\
l'informatique en général, parce que sans \\
quoi, c'est vraiment un méli-mélo ... \\
\end{tabular} & $\begin{array}{l}\text { backlog } \\
\text { phrase }\end{array}$ & $\begin{array}{l}\text { textuel } \\
\text { (relations } \\
\text { thématiques } \\
\text { backlog/ } \\
\text { Compaq) }\end{array}$ \\
\hline 22 & $\begin{array}{l}\text { Lit, cherche } \\
\text { dans contexte } \\
\text { renforcement } \\
\text { de l'hypothèse } \\
\text { établie en } 19\end{array}$ & $\begin{array}{l}\text { d'autant plus que, dans le paragraphe } \\
\text { suivant, il revient «older Deskpro lines have } \\
\text { produced record sales ... branched out ... } \\
\text { and this fall brought out Prolineas to Japan». }\end{array}$ & article & $\begin{array}{l}\text { intratextuel : } \\
\text { (relations } \\
\text { thématiques } \\
\text { backlog/ } \\
\text { Compaq) }\end{array}$ \\
\hline 23 & $\begin{array}{l}\text { Explique } \\
\text { texte }\end{array}$ & $\begin{array}{l}\text { Donc, on est en train de parler de } \\
\text { l'entreprise Compaq elle-même, et on est en } \\
\text { train de parler du carnet de commandes ... }\end{array}$ & $\begin{array}{l}\text { order backlog } \\
\text { article }\end{array}$ & $\begin{array}{l}\text { intratextuel } \\
\text { (relations } \\
\text { thématiques) }\end{array}$ \\
\hline 24 & $\begin{array}{l}\text { Inférence, } \\
\text { explique }\end{array}$ & $\begin{array}{l}\text { Donc, c'est pas possible que ce soit des ... } \\
\text { et, en plus, c'est même pas un retard de } \\
\text { livraison ... C'est qu'ils ont le carnet rempli } \\
\text { pour trois mois, enfin, qu'ils ont trop de ... }\end{array}$ & $\begin{array}{l}\text { order backlog } \\
\text { contexte } \\
\text { pragmatique }\end{array}$ & $\begin{array}{l}\text { extraling. } \\
\text { (logique des } \\
\text { mondes } \\
\text { possibles) } \\
\end{array}$ \\
\hline 25 & $\begin{array}{l}\text { Applique à } \\
\text { sa traduction } \\
\text { le résultat } \\
\text { de l'analyse } \\
\text { en } 19-24\end{array}$ & $\begin{array}{l}\text { Oui, je vais inverser ... } \\
\text { [Le répondant place la proposition } \\
\text { concernant la livraison des commandes } \\
\text { avant celle concernant la baisse des prix } \\
\text { dans le secteur.] }\end{array}$ & phrase traduite & $\begin{array}{l}\text { procédural } \\
\text { (transfor- } \\
\text { mation de } \\
\text { l'organicité } \\
\text { textuelle) }\end{array}$ \\
\hline 26 & $\begin{array}{l}\text { Analyse } \\
\text { liens de } \\
\text { dépendance } \\
\text { dans contexte, } \\
\text { exprime son } \\
\text { mécontente- } \\
\text { ment } \\
\end{array}$ & $\begin{array}{l}\text { Alors, voyons ... a connu un succès } \\
\text { foudroyant, hum ... Cette histoire de carnet } \\
\text { de commandes, ça me casse les pieds } \\
\text { «cutting market leader», bon, non, } \\
\text { «aggressively priced». Bon, là, s'ils ont un } \\
\text { retard de trois mois, c'est qu'ils ont trop } \\
\text { d'ordres en tous cas ... }\end{array}$ & $\begin{array}{l}\text { order backlog } \\
\text { contexte }\end{array}$ & $\begin{array}{l}\text { intratextuel } \\
\text { (relations } \\
\text { thématiques) }\end{array}$ \\
\hline 27 & Traduit & $\begin{array}{l}\text { Donc, a connu un succès fulgurant, } \\
\text { deux points. }\end{array}$ & phrase traduite & syntagmatique \\
\hline 28 & $\begin{array}{l}\text { Identifie } \\
\text { probl. non } \\
\text { réglé } \\
\end{array}$ & $\begin{array}{l}\text { Bon, je vais revoir ce backlog ... Je suis pas } \\
\text { du tout convaincue }\end{array}$ & backlog & procédural \\
\hline 29 & $\begin{array}{l}\text { Cherche en } \\
\text { vain backlog } \\
\text { dans Robert } \\
\& \text { Collins }\end{array}$ & ...Évidemment ... & backlog & lexical \\
\hline
\end{tabular}




\begin{tabular}{|l|l|l|l|l|}
\hline & $\begin{array}{l}\text { Nature de } \\
\text { l'opération }\end{array}$ & Protocole de verbalisation & Locus / Focus & $\begin{array}{l}\text { Niveau } \\
\text { d'analyse }\end{array}$ \\
\hline 30 & $\begin{array}{l}\text { Consulte } \\
\text { dictionnaire }\end{array}$ & $\begin{array}{l}\text { Voyons à order, j'avais pas vu tout à } \\
\text { l'heure ... «Order», disposition, séquence } \\
\text { OK, j'm'en fiche ... good order, en ordre, in } \\
\text { order to do, bon, ça je m'en fiche ... peace } \\
\text { control, on ... architecture ... ordre, } \\
\text { commandement, consigne ... that's an order, } \\
\text { OK ... OK, commande, commercial, voilà, } \\
\text { to order, to give, to place, the shelves, on } \\
\text { order, to do something to order, je m'en } \\
\text { fous, order book, carnet de commandes, } \\
\text { the company's order books were full, voilà, } \\
\text { c'est bien ce qui me semblait. Order form, } \\
\text { bon de commande, order paper, ordre du } \\
\text { jour, command, ça c'est verbe déjà ! }\end{array}$ & $\begin{array}{l}\text { lexico- } \\
\text { sémantique }\end{array}$ & order \\
\hline 31 & $\begin{array}{l}\text { Applique } \\
\text { résultat de } \\
30 \text { à } \\
\text { explication }\end{array}$ & $\begin{array}{l}\text { C'est effectivement, alors, un retard dans ... } \\
\text { dans l'exécution des commandes, ça doit } \\
\text { être ça ... }\end{array}$ & backlog & $\begin{array}{l}\text { lexico- } \\
\text { sémantique }\end{array}$ \\
\hline 32 & $\begin{array}{l}\text { Paraphrase } \\
\text { Un retard donc, provoquant un retard de } \\
\text { trois mois dans l'exécution ... }\end{array}$ & $\begin{array}{l}\text { 3-month order } \\
\text { backlog }\end{array}$ & syntagmatique \\
\hline $\begin{array}{l}\text { Révise } \\
\text { cooccurrents } \\
\text { lexicaux }\end{array}$ & $\begin{array}{l}\text { si tant est qu'on exécute un carnet de } \\
\text { commandes ... oh ! la livraison des } \\
\text { commandes et ça ira ! }\end{array}$ & $\begin{array}{l}\text { collocations } \\
\text { verbales de } \\
\text { commande }\end{array}$ & phraséologique \\
\hline
\end{tabular}

\subsection{Les analyses de processus comme modèle de description traductologique}

\subsubsection{Portée}

Cette analyse éclaire l'activité traduisante sous différents aspects. Nous nous contenterons de quelques observations regroupées en trois points.

Mise en évidence des étapes constitutives du processus de traduction. En regardant l'ensemble de ces 33 opérations (dont plusieurs auraient pu être décomposées en de plus petites unités), on voit qu'elles se regroupent en quatre grandes catégories : la compréhension, l'expression dans la langue d'arrivée, l'insertion du sujet dans le processus et le transfert linguistique (ou traduction proprement dite).

- Le sens se construit par approximations. Le sens de l'expression three-month order backlog s'élabore au cours d'opérations de différentes natures visant la définition du concept (consultation du dictionnaire, paraphrase explicative telles qu'en $1,4,6,16)$ ou visant l'application de la définition du concept au contexte discursif et pragmatique $(21,22,23,24)$.

- $\underline{\text { La formulation de la traduction comporte des opérations de recherche à }}$ plusieurs niveaux. Le répondant effectue les opérations logiques pour rétablir les structures syntaxico-sémantiques profondes. Il cherche, de 18 à 24 , à répondre à la question : à quoi se rapporte le «three-month order backlog», à l'industrie ou à la société Compaq? Dans la réécriture de surface (en 25), il effectue les transformations nécessaires. Il teste aussi différents mots pour trouver la collocation de commande la plus juste qui soit : exécuter, remplir, livrer, faire passer (en 14, 15, 16 et 33). 
- Le sujet s'insère dans le processus. Il exprime ses réactions face à sa réception du texte source et à son traitement du texte cible. Soit il exprime une réaction émotive primaire $(2,12)$; soit il identifie un problème et réévalue sa démarche $(3,5,8$, $10,25,28$ et partiellement 30 ).

- Traduction. Elle se fait par écrit, avec retours en arrière, ratures et réécriture.

Cette classification par catégories d'opérations rejoint les grilles utilisées dans les travaux déjà cités. La récurrence de ces opérations, ainsi que le consensus des observateurs sur leur reconnaissance et leur identification (quelles que soient les étiquettes) assurent la possibilité de reproduire de telles analyses.

Rendement du protocole de verbalisation. Il est remarquable qu'un seul segment de phrase nous ait donné autant de renseignements sur l'activité mentale du sujet face à un problème de compréhension. Cette analyse confirme aussi la présence et le rôle de certaines composantes et de leurs relations, notamment aux niveaux lexical, syntaxique et sémantique, comme, par exemple, en 18,19,21-24. Elle renforce la validité des explications apportées par les théories actancielles (Fillmore 1968 ; Melčuk 1978) aux modalités de désambiguïsation, et qui aboutissent, ici, à une inversion de l'ordre des propositions de la phrase (25), une fois les relations thématiques solidement comprises.

Non-linéarité du processus de traduction sur l'axe temporel. Même si les opérations se suivent dans le temps, la résolution des problèmes décrit des va-et-vient qui donnent lieu à des réponses provisoires, remises en question ou maintenues à des étapes ultérieures. Par sa présentation, la grille d'analyse qui reproduit les étapes du processus, est nécessairement linéaire. Elle ne met pas suffisamment en relief l'interaction entre les étapes de la compréhension et la formulation de la traduction. On peut corriger ce problème en adoptant le modèle de la double hélice dont nous faisons une illustration ci-dessous pour quelques étapes seulement (opérations 1, 13-14, 24-25 et 33), les contraintes d'espace nous forçant à présenter de façon discontinue la chaîne des opérations.

Le graphe a pour objet de mettre en regard l'un de l'autre le processus de compréhension (la courbe foncée) et le processus de traduction (la courbe claire).

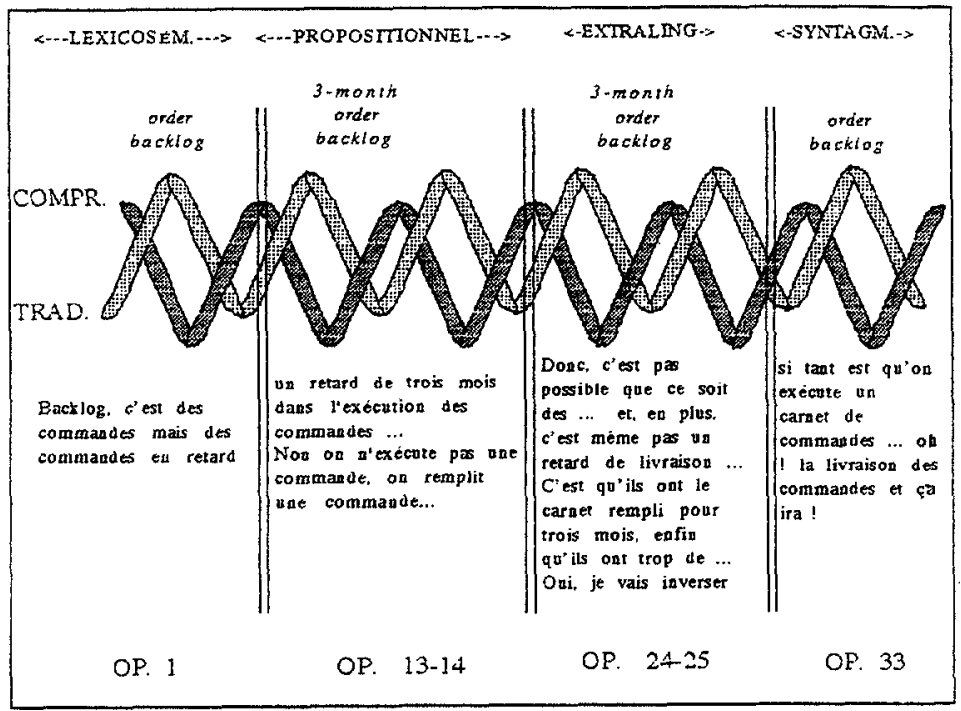

Modèle de la double hélice 
Au cours de l'opération 1, on constate la correspondance entre le travail d'analyse lexicosémantique et la formulation d'une première équivalence lexicale. Au cours de l'opération $24-25$, le raisonnement conduit le répondant à rejeter l'idée que le délai de trois mois dans l'exécution des commandes se rapporterait à l'industrie, et au niveau de sa traduction, à inverser l'ordre des propositions (comme on le voit dans les différentes corrections que le répondant apporte à sa traduction écrite). Entre les opérations $13-14$ et 33 (dernière opération consacrée à l'expression order backlog), il n'y a pas de différence au niveau de la traduction (la même ambiguïté subsiste à cause de l'emploi de retard au lieu de délai). Pourtant, on sait tout le travail d'analyse qui a été effectué permettant au répondant d'approfondir sa compréhension textuelle et pragmatique.

\subsubsection{Limites}

Dans une perspective d'analyse descriptive où la méthodologie occupe une place très importante, l'analyse des processus soulève au moins trois grands problèmes.

Premièrement, on est obligé d'exercer des contraintes et même de prendre des décisions arbitraires par rapport à la segmentation nécessaire d'un processus mental qui est fait non pas d'entités discrètes mais d'un continuum, et cela sans compter le fait que la verbalisation des opérations épouse la linéarité du discours et ne laisse pas de place à l'expression ou à la reconnaissance d'opérations mentales simultanées. De façon concrète, l'analyste se trouve parfois aux prises avec des problèmes de brouillage des frontières et l'on doit, alors, procéder par hypothèse.

Deuxièmement, l'interprétation du protocole quant à la nature des opérations et au statut des phénomènes observés dépend grandement des hypothèses de recherche. En ce qui nous concerne, par exemple, il nous semble logique de privilégier les opérations qui nous aident à mieux expliquer la compréhension, étant donné l'importance qu'occupe, dans notre conception de la traduction, l'analyse sémantique à quelque niveau que ce soit. Le corollaire négatif, c'est que l'intrusion du sujet avec ses réactions émotives est enregistrée comme donnée brute mais est évacuée dans un modèle plus abstrait, mais réductionniste, tel que celui de la double hélice.

Troisièmement, la faiblesse la plus marquée, à notre avis, réside dans le peu de fiabilité du protocole en tant que modèle d'élicitation. Il y a une trop grande variabilité de la capacité de verbaliser d'un sujet à l'autre. Même chez les sujets qui le font bien, les contenus sont de nature et d'intérêt très variés. Rien ne nous dit que les opérations mentales les plus importantes soient exprimées spontanément. Bien sûr, on essaie de combler cette lacune par les questionnaires et les entrevues, mais, même là, il n'est pas certain que ces opérations puissent surgir à la conscience du répondant. Dans de nombreuses recherches on pallie ce problème en formant les répondants à la méthode de verbalisation, mais ce faisant, non seulement on a perdu l'ingénuité des réponses du locuteur naïf, mais on risque même de créer des artefacts puisque les témoins (expérimentés ou formés) seront peut-être amenés, sans mauvaise intention, à dire ce que nous voulons entendre.

Ces critiques ne signifient pas que l'analyse des processus perd son intérêt au plan descriptif, bien au contraire, mais on interprète mieux les résultats quand on connaît les limites de l'expérience.

\subsection{Les analyses de processus comme catalyseur d'une problématique de la traductologie}

D'un point de vue épistémologique, l'analyse des processus provoque la réflexion sur quelques problèmes fondamentaux dans les théories de la traduction sur lesquels on ne s'est peut-être pas suffisamment arrêté. Nous nous limiterons dans ce débat à trois questions qui ne sont pas complètement indépendantes les unes des autres. Il s'agit 1) de 
la finalité de la recherche théorique en traductologie ; 2) de l'explication des phénomènes par élargissements successifs des perspectives, du linguistique au monde des connaissances ; 3) de la prédictibilité des modèles théoriques. Nous discuterons surtout des deux premiers problèmes.

\subsubsection{Finalité de la recherche théorique}

On peut commencer par poser une simple question : quel est le but ultime de la démarche théorisante en traductologie? Question subsidiaire: en quoi l'analyse des processus s'insère-t-elle dans cette même finalité ? La réponse à la première question peut paraître évidente, mais elle n'est pas toujours exprimée dans les recherches. Il s'agit essentiellement de mieux comprendre le déroulement de l'activité traduisante afin de mieux la diriger, soit pour soi-même soit dans un contexte pédagogique, pour la formation des traducteurs et des interprètes. Dans ce cas, l'analyse des processus peut être en parfaite concordance avec la recherche théorique (Pöchhacker 1992; Kussmaul 1995). Elle peut faire voir les conditions de succès et le rendement d'un certain nombre de stratégies qu'il est possible de recueillir et d'organiser à des fins d'enseignement ou d'apprentissage. Les théories structuralistes en traductologie, comme le polysystème de Toury (1980) et le modèle intégratif de House (1977) ou de Larose (1989) se situent, à notre avis, dans une perspective fonctionnaliste d'où la finalité pragmatique n'est pas absente ( $c f$. le «contrat de traduction» de Larose) ; cependant, dans ces dernières, la visée pragmatique est moins contraignante. Notons, toutefois, que toute visée pragmatique lie l'intérêt d'une théorie à ses applications (résultats réels) et, à la limite, pourrait fausser l'orientation de la recherche théorique.

À l'autre extrême, on peut parler d'une théorie de la traduction tout à fait indépendante des préoccupations utilitaires de quelque nature que ce soit. On est alors dans la recherche d'explications seulement pour le plaisir ou le désir de trouver une réponse satisfaisante à des questions touchant le langage en général comme phénomène social, ou même le comportement humain tout court, l'activité traduisante n'étant que prétexte ou point de départ à une problématique appelée assez rapidement à la dépasser (Berman 1985 ; Brisset 1990). Dans certains cas, les mêmes thèmes ou les mêmes préoccupations peuvent conduire à des démarches et à des résultats diamétralement opposés à ceux des recherches à des fins pédagogiques (ex. : la fidélité, l'équivalence, le sens, l'adaptation, l'erreur, etc.).

Avec ses forces et ses faiblesses, l'analyse des processus peut servir ou handicaper les deux perspectives. Dans un cas, le chercheur s'intéressera davantage à dégager les stratégies conduisant à des résolutions de problèmes; la finalité pragmatique peut alors devenir un critère pour décider de la pertinence des données à exploiter. Le risque est de court-circuiter les opérations qui ne semblent pas créer de problème pratique, indépendamment de leur importance logique, et d'accorder un poids démesuré à des difficultés de traduction parfois superficielles.

Dans l'autre cas (théories plus générales), le chercheur s'intéressera davantage à la valeur globale, «holistique», de la démarche. Selon la problématique qui le préoccupe, il appuiera sans doute sa démonstration sur la nature heuristique des interventions du sujet traduisant : conscience qu'il a des gestes posés, justification de ses choix, effets de ses réactions affectives ou de sa capacité mémorielle. L'analyse des processus offre aussi l'occasion de reprendre le débat sur l'articulation entre les champs référentiels (virtuels, vastes, plus ou moins organisés) et l'actualisation d'une signification, par décision du traducteur, en un temps donné et à un point quelconque du discours (Beaugrande 1980). L'inconvénient, c'est que toute approche globalisante, pour être crédible, exigerait un appareil scientifique d'explication et de vérification trop coûteux. On revient alors au problème de la pertinence qui se pose chaque fois que l'on manipule des données. 


\subsubsection{Explications par élargissements des perspectives}

Parler des champs virtuels de connaissances nous amène à un extrême dans l'ordre scientifique organisé, celui des mondes possibles, de nature essentiellement spéculative. Cet ordre des mondes possibles est bel et bien présent dans l'imagination, la pensée et le langage (Alice au pays des merveilles, métaphysique, poétique).

D'un autre côté, nous avons l'ordre des plus petits éléments concrets du monde signifiant (ex. : morphème). Ces éléments, organisés en sous-ensembles successifs, ont une certaine matérialité (substance) et une forme : un mot est un mot, se prononce, s'écrit, se distingue d'un autre mot de la même langue, a un ou plusieurs sens reconnus par des locuteurs natifs même hors contexte, et se traduit. Or, le problème soulevé par la compréhension du texte à traduire s'insère nécessairement dans une démarche de contextualisation progressive, par recours à des contextes éventuellement de plus en plus larges pour expliquer parfois une seule unité. C'est le cas, dans l'exemple que nous avons développé, de backog où pratiquement tous les contextes ont été explorés. On peut formaliser la hiérarchie entre ces contextes de différentes façons : boîtes, diagrammes inclusifs, comme dans la théorie du «skopos» de Reiss et Vermeer (1984) ou dans les modèles théoriques que nous avons appelés intégratifs. Le recours au contexte implique différents niveaux d'analyse en interaction les uns avec les autres :

$$
\begin{gathered}
\text { LEXICAL <-> MORPHOLOGIQUE <-> MORPHOSYNTAXIQUE <-> } \\
\text { SYNTAXIQUE <-> TEXTUEL <-> HYPERTEXTUEL <-> PRAGMATIQUE } \\
<->\text { CHAMP ORGANISÉ DES CONNAISSANCES <-> MONDES POSSIBLES }
\end{gathered}
$$

La solution des problèmes sémantiques à un point quelconque d'un texte fait souvent appel à des données d'autres niveaux, supérieurs ou inférieurs dans la chaîne, qui apportent chacun un éclairage - ou des contraintes - qui permettent de construire le sens ou de lever l'ambiguïté.

L'analyse des protocoles présentée ci-dessus nous a permis de montrer que le répondant a eu recours à presque tous ces niveaux. Il a répondu aux questions qu'il se posait, mais sa traduction n'est pourtant pas tout à fait satisfaisante. La notion de retard n'est pas la même chose que celle de délai, la première impliquant un manquement par rapport à un engagement. Du point de vue de la réalité commerciale de l'entreprise, la différence est énorme.

D'un point de vue théorique, le recours à l'explication par contextes soulève deux problèmes très sérieux. Premièrement, on peut s'interroger sur la cohérence des règles qui gouvernent les liens d'interdépendance et de hiérarchie entre les contextes. Puisqu'on peut naviguer autant vers les niveaux inférieurs que vers les niveaux supérieurs, il est quasiment impossible de déterminer ce qui déclenche l'appel au contexte, autrement que par l'insatisfaction du sujet ( $c f$. le seuil d'inconfort de Gile, 1990) ou sa curiosité, le tout relevant d'une conception fonctionnaliste. Or, comme ces facteurs varient qualitativement et quantitativement d'un sujet ou d'une situation à l'autre, on ne peut pas, au moins dans une perspective théorique, parier de système de règles. Pour ce même texte, d'autres sujets au cours de la même expérience ont traduit vite, mais mal, sans se poser de questions, d'autres vite et correctement, avec un minimum de questions.

Qui plus est, il peut y avoir une contradiction entre les informations reçues à différents niveaux. Dans ce cas, les stratégies oscillent, en fonction d'un calcul de risques, entre l'interprétation conformément au possible ou au plausible et le transcodage (interprétation littérale). Mais, s'il n'y a pas de contradiction et que le niveau de représentation lexicale par exemple suffit, pourquoi recourir à des niveaux contextuels supérieurs, plus englobants? C'est le principe du moindre effort (Krings 1986) et c'est cohérent avec 
les positions de Jackendoff (1985) ou de Langacker (1987) selon lesquelles le cognitif peut s'intégrer à tous les niveaux linguistiques touchés par la sémantique. Kleiber (1994) adopte un point de vue plus nuancé. Dans un bilan sur le calcul classique du sens en linguistique et l'approche cognitive, il reconnaît que cette dernière constitue un progrès indéniable, même si elle a beaucoup de faiblesses au plan de la représentation formelle des contextes. Et c'est là que se pose le deuxième problème.

D'un point de vue formel, on peut ou on doit considérer les divers niveaux contextuels comme autant de systèmes, à la fois distincts et interdépendants. Or, on doit admettre que les systèmes aux niveaux linguistique et même textuel reposent sur un grand nombre de principes communs et ne sont pas construits sur les mêmes paradigmes que les modèles de représentations de connaissances. Ils n'empruntent pas, non plus, les voies habituelles d'accès aux sphères sociales, culturelles, voire «cosmiques» (Bohm et Peat 1990).

L'articulation entre ces deux ensembles est le cœur de notre interrogation: Sommes-nous dans un cas de rupture épistémologique? D'un côté, différentes étapes du processus peuvent recevoir une explication formelle dans le cadre d'une théorie linguistique ou d'une grammaire de texte. La formalisation peut être même assez poussée pour qu'elle soit en grande partie automatisée (ex. : le projet TAUM, Isabelle 1986). D'un autre côté, lorsqu'on pense à des textes dont la compréhension s'appuie davantage sur des champs très larges de connaissances (culture) et les mondes possibles, on touche aux limites d'une approche formelle ou même fonctionnelle de la compréhension et du sens. Il y a parfois des solutions ad hoc, soit en principe par extension des frontières de la lexico-sémantique, soit en pratique par enrichissement des bases de données dictionnairiques et terminologiques. Dans l'exemple du protocole retenu, la suite des processus aurait été différente si, à l'étape 15 , notre répondant avait consulté le dictionnaire de langue commerciale qui était à sa disposition. Quelle que soit l'ingéniosité de ces solutions, la cohérence logique et la systématicité des stratégies ne peuvent pas être établies. Toute tentative de formalisation reste boîteuse car aucune théorie ne permet à ce jour d'intégrer épistémologiquement ces différents niveaux. À défaut de références à un système formel intégré, la tentation est grande de recourir à d'autres disciplines pour mieux expliquer la compréhension (philologie, herméneutique, philosophie, psychanalyse). Mais alors, il faut se demander dans quelle mesure on est encore en traductologie.

\subsubsection{Prédictibilité des modèles théoriques}

Certains penseurs et historiens des sciences ont avancé l'idée que les théories scientifiques, à la différences des sciences descriptives ou taxinomiques, devaient avoir une capacité de prédiction. Nous ne souscrivons pas forcément à cette perspective dichotomique en ce qui concerne les sciences humaines, mais nous la retenons ici pour les besoins de la discussion. Une théorie traductologique qui s'appuierait sur l'analyse des processus posséderait-elle la propriété prédictive ? C'est un débat assez important pour être traité en profondeur ; nous n'avons pas la prétention de le faire ici.

Pour poser le problème en termes simples, on devrait pouvoir établir que, si un sujet applique convenablement telle suite d'opérations, de règles et de principes, il devrait comprendre et traduire correctement, au moins pour l'essentiel. Or, il n'en est rien. En corollaire, la non-application de ces opérations ou de ces stratégies, et la violation en tout ou en partie de ces règles, devraient conduire à l'échec, ce qui n'est nullement vérifié puisqu'on peut même, dans une certaine mesure, traduire sans comprendre. Le défaut de prédictibilité entraîne des conséquences sérieuses et immédiates dans le cas d'une théorie traductologique à visée utilitaire. Il peut sembler moindre dans le cadre d'une théorie spéculative puisque cette incapacité peut, paradoxalement, ne pas être considérée comme 
un problème mais plutôt comme un postulat. Quelle que soit la théorie, on ne peut éviter de s'interroger sur l'exigence de la prédictibilité.

\section{CONCLUSION}

Cette réflexion sur l'analyse des protocoles de traduction nous a permis de postuler, en traductologie, l'interdépendance entre les modèles expérimentaux et descriptifs, d'une part, et les tentatives de théorisation, d'autre part. Les approches expérimentales doivent être poursuivies, ne serait-ce que pour valider les théories traductologiques à finalité pragmatique. En effet, le progrès des recherches serait plus rapide s'il y avait plus de suivi et de concertation entre les chercheurs pour assurer la répétition des expériences, la compilation systématique des données, la validation des corrélations et des indices, en somme, tout ce qui fait la force des méthodes quantitatives.

D'un point de vue historique, les chercheurs ont raison d'éviter l'analogie avec l'évolution d'autres sciences, surtout des sciences physiques et naturelles, et de refuser la distinction catégorique entre un stade préscientifique et un stade postérieur, théorique. Les recherches expérimentales, qualitatives et quantitatives, sont des conditions nécessaires au progrès de la traductologie. Nous sentons néanmoins le besoin de rechercher des modèles explicatifs ayant un plus grand pouvoir de formalisation et d'abstraction. En effet, les données recueillies par une démarche comme celle des protocoles verbaux sont infiniment riches mais charrient aussi, autant pour le témoin que pour l'analyste, tous les pièges de la phénoménologie.

En ce qui concerne l'analyse des protocoles, la relation établie ou postulée entre la pertinence des stratégies et l'atteinte des objectifs de compréhension en traduction ne facilite pas les choses. En effet, un calcul empirique qui donnerait de bons résultats n'est pas forcément une garantie d'explication scientifique. En observant que le soleil tourne autour de la terre, on peut parvenir à bien s'orienter ou à dire l'heure qu'il est, mais on est loin de la vérité scientifique. Or, les protocoles restent dans l'ensemble une entreprise fortement empirique qui peut conduire à de fausses explications. Néanmoins, il faut reconnaître, comme démarche heuristique, le fort rendement des analyses sur les processus. Elles embrassent et définissent aisément les frontières des modèles de description, même les plus intégratifs, puisqu'elles peuvent couvrir tout l'espace traductif, de la première opération sur le texte source à la dernière sur le texte cible. C'est aussi pour cette raison qu'il est difficile d'imaginer la construction d'une théorie globale en traductologie, quelle qu'en soit la perspective, et a fortiori dans une perspective cognitiviste, qui éviterait de rendre compte des problèmes, des stratégies et des décisions révélées par l'étude des processus. Le protocole est donc un outil qui n'a pas son équivalent dans l'histoire de la recherche en traductologie.

Note

1. Nous remercions Danielle Collignon pour son aide technique, ainsi que les arganismes FCAR et CRSH pour leur soutien financier.

\section{RÉFÉRENCES}

BEAUGRANDE, R. de (1980) : Factors in a Theory of Poetic Translating, Assen, Van Gorcum.

BÉLANGER, G. (1992): Étude des relations cohésives grammaticales: perspectives traductologique et typologique, thèse de doctorat en linguistique, Université de Sherbrooke.

BERMAN, A. (1985) : «La traduction et la lettre ou l'auberge du lointain», Les tours de Babel, Mauvezin, Trans-Europ-Repress.

BOHN, D. \& F. D. PEAT (1990) : La conscience et l'univers, Monaco, Éditions Du Rocher.

BRISSET, A. (1990): Sociocritiqué de la traduction, théâtre et altérité au Québec, Longueuil, Québec, Éditions le Préambule.

CHALMERS, A. (1987): Qu'est-ce que la science?, Paris, Éditions La Découverte. Original anglais : What is this Thing Called Science? An Assessment of the Nature and Status of Science and its Methods.

DANCETTE, J. (1994) : «Comprehension in the Translation Process: An Analysis of Think-Aloud Protocols», 
C. Dollerup and A. Lindegaard (Eds.), Teaching Translation and Interpreting: Insights, Aims, Visions, Selected papers from the Second "Language International" Conference, Elsinore, Denmark, 4-6 June 1993, Amsterdam \& Philadelphia, John Benjamins.

DANCETTE, J. (1995) : Parcours de traduction ; Analyse expérimentale des processus de compréhension, Lille, Presses Universitaires de Lille.

DANCETTE, J. (à paraitre) : «Analyzing Comprehension Processes in Translation», J. Danks \& G. Shreve, Cognitive Processes in Translation and Interpretation, Sage Publication, Kent Psychology Forums.

DECHERT, H. W. (1987) : «Analyzing Language Processing through Verbal Protocols», Introspection in Second Language Research, Faerch and Kasper (Eds.), Introspection in Second Language Research, Clevedon, Multilingual Matters.

DECHERT, H. W. and U. SANDROCK (1986) : «Thinking-Aloud Protocols ; the Decomposition of Language Processing», V. Cook (Ed.), Experimental Approaches to Second Language Learning, Oxford, Pergamon.

DEMERS, G. (1992) : «Les statistiques au service de la pédagogie de la traduction», Traduction, terminologie et rédaction (TTR), 5-1.

DUPONT, S. (1993): De la dynamique de la traduction ; étude empirique des stratégies de compréhension, mémoire de maîtrise, Université de Montréal.

FILLMORE, C. J. (1968) : «The Case for Case», Bach and Harms, Universals in Linguistic Theory, New York, Holt Rinehart.

FILLMORE, C. J. (1976) : «Frame Semantics and the Nature of Language», Harnad, S. R., Steklis, H. D. \& Lancaster, J. (Eds.), Origins and Evolution of Language and Speech, New York, The New York Academy of Sciences.

GERLOFF, P. (1987) : «Identifying the Unit of Analysis in Translation: Some Uses of Think-Aloud Protocol Data», Faerch, Claus et Gabriele Kasper (Eds.), Introspection in Second Language Research, Clevedon, Multilingual Matters.

GERLOFF, P. (1988) : From French to English: A Look at the Translation Process in Students, Bilinguals, and Professional Translators, Ph.D thesis, Harvard University.

GILE, D. (1990) : «La traduction et l'interprétation comme révélateurs des mécanismes de production et de compréhension du discours», Meta, 35-1.

GILE, D. (1991) : «Methodological Aspects of Interpretation and Translation Research», Target, 3-2.

GRANT, P. (1992) : The Integration of Theory and Practice in the Development of Summary-Writing Strategies, $\mathrm{Ph} . \mathrm{D}$ thesis, Département de linguistique et de traduction, Université de Montréal

HARTMAN, D. (1991): Eight Readers Reading; The Intertextual Links of Able Readers Using Multiple Passages, Ph.D thesis, University of Pittsburgh (Penn.).

HOUSE, J. (1977) : A Model for Translation Quality Assessment, Tübingen, TBL, Verlag Gunter Narr.

ISABELLE, P. (1986) : «Le projet TAUM de traduction automatique», Revue québécoise de linguistique, 5-2.

JÄÄSKELÄINEN, R. (1993) : «Recent Trends in Empirical Translation Research», S. Tirkkonen-Condit and J. Laffling (Eds.), Kielitieteellisiäa Tutkimuksia/Studies in Languages, Joensuu, University of Joensuu, Faculty of Arts.

JACKENDOFF, R. (1985) : Semantics and Cognition, Cambridge, The MIT Press.

KLEIBER, G. (1994): «Contexte, interpretation et mémoire: approche standard vs approche cognitive», Langue française. Le lexique : construire l'interprétation, Larousse, $\mathrm{n}^{\circ} 103$.

KRINGS, H. P. (1986) : Was in den Köpfen von Übersetzern Vorgeht, Tübingen, Gunter Narr.

KRINGS, H. P. (1988): «Blick in die "Black Box" - Eine Fallstudie zum Übersetzungsprozess bei Berufsübersetzern», Arntz (Ed.), Textlinguistik und Fachsprache. Akten des internationalen übersetzungswissenschaftlichen AlLA Symposions, Hildesheim, Olms.

KUSSMAUL, P. (1995) : Training the Translator, Amsterdam / Philadelphie, John Benjamins.

LANGACKER, R. (1987) : Foundations of Cognitive Grammar (vol. 1), Stanford, Stanford University Press.

LAROSE, R. (1989): Théories contemporaines de la traduction, Presses de l'Université du Québec.

LÖRSCHER, W. (1991) : Translation Performance, Translation Process and Translation Strategies, Tübingen, Gunter Narr

LÖRSCHER, W. (1992): «Process-oriented Research into Translation and Implications for Translation Teaching», Traduction, terminologie et rédaction (TTR), 5-1.

MEL'ČUK, I. (1978) : «Théorie de langage, théorie de traduction», Meta, 23-4.

MÉNARD, N. (1983) : Mesure de la richesse lexicale, Genève, Paris, Slatkine, Champion.

MINSKY, M. (1974) : A Framework for Representing Knowledge, Memo 306, Cambridge, The MIT Press.

MONDAHL, M. et K. A. JENSEN (1992) : «Information Processing in a Translation Task», Multilingua, 11-2.

MULLER, C. (1973) : Initiation aux méthodes de la statistique linguistique, Paris, Hachette.

PÖCHHACKER, F. (1992): «The Role of Theory in Simultaneous Interpreting», C. Dollerup and A. Lindegaard (Eds.), Teaching Translation and Interpreting: Training. Talent and Experience, Selected papers from the First "Language International" Conference, Elsinore, Denmark, Amsterdam / Philadelphia, John Benjamins. 
RAYNER, K. et al. (1989) : «Eye Movements and On-line Language Comprehension Processes», Language and Cognitive Processes, 4-3/4.

REISS, K. \& H. VERMEER (1984): Grundlegung einer Allgemeinen Translationstheorie, Tübingen, Max Niemeyer.

SANDROCK, U. (1982): Thinking-Aloud Protocols (TAPs) _Ein Instrument zur Dekomposition des komplexen Prozesses «Übersetzen», Habilitationsschrift, Universität Kassel.

SCHÄFFNER, C. (1993) : «Meaning and Knowledge in Translation», Y. Gambier and J. Tommola (Eds.), SSOTT IV Translation and Knowledge, University of Turku, Grafia Oy, Finland.

SÉGUINOT, C. (1989) : The Translation Process, Toronto, School of Translation, York University.

SÉGUINOT, C. (1990) : «Interpreting Errors in Translation», Meta, 35-1.

SELESKOVITCH, D. et M. LEDERER (1984) : Interpréter pour traduire, Paris, Didier.

TIRKKONEN-CONDIT, S. (1992): «The Interaction of World Knowledge and Linguistic Knowledge in the Processes of Translation. A Think-Aloud Protocol Study», Lewandowska-Tomaszczyk et Thelen (Eds.), Translation and Meaning, Part 2, Rijkshogeschool Maastricht, Faculty of Translation and Interpreting.

TOURY, G. (1980) : In Search of a Theory of Translation, Tel Aviv, The Porter Institute for Poetics and Semiotics.

WHALEN, K. \& N. MÉNARD (1995) : «L1 and L2 writers strategic and linguistic knowledge - A model of multiple-level discourse processing», Language Learning, 45-3.

\section{ANNEXE}

\section{COMPAQ}

How it Made its Impressive Move out of the Doldrums

Eckhard Pfeiffer is a happy man these days, and it shows. In only a year as president, he has managed to restore the glitz to Compaq Computer Corp. Once the victim of price wars, Compaq is now the aggressor. Its low-cost ProLinea pcs, introduced last June, were an immediate hit, spurring industrywide price cuts and generating a three-month order backlog. In the company's older Deskpro lines, new models and lower prices have produced record sales. In August, Compaq branched out into laser printers and this fall brought ProLineas to Japan, undercutting market leader nec's prices by $50 \%$. Then it extended the price battle in the U.S. with cutrate "servers" for pc networks.

No wonder Pfeiffer is happy. But not, repeat not, complacent. On the eve of reporting third-quarter earnings - the first to truly show the payoff of the new strategy - he sprang his October surprise: price cuts of as much as $32 \%$ across Compaq's already aggressively priced product line. The move, which Compaq said reflected the economies of scale it's achieving with its new high-volume strategy, brought its cheapest machine down to $\$ 799$ and is bound to provoke more price cuts. It also came just in time to upstage ibm's announcement of its belated entry into the bargain pc business (page 152). "I know that everybody out there wants to be where we are" says Pfeiffer.

Business Week, November 2, 1992, p. 146. 